\title{
Green's Function Solution of Non-Homogenous Regular Sturm-Liouville Problem
}

\author{
Abdelgabar Adam Hassana* \\ Department of Mathematics, College of Science and Arts at Tabrjal, AlJouf University, Kingdom of Saudi Arabia
}

\begin{abstract}
In this paper, we propose a new method called $\exp (-\phi(\xi))$ fractional expansion method to seek traveling wave solutions of the nonlinear fractional Sharma-Tasso-Olver equation. The result reveals that the method together with the new fractional ordinary differential equation is a very influential and effective tool for solving nonlinear fractional partial differential equations in mathematical physics and engineering. The obtained solutions have been articulated by the hyperbolic functions, trigonometric functions and rational functions with arbitrary constants.
\end{abstract}

\section{Introduction}

The series solution of differential equation yields an infinite series which often converges slowly. Thus it is difficult to obtain an insight into over-all behavior of the solution [1,2]. The Green's function approach would allow us to have an integral representation of the solution instead of an infinite series.

To obtain the filed $y$, caused by distributed source we calculate the effect of each elementary portion of source and add (integral) them all. If $G\left(r, r_{0}\right)$ is the field at the observers point $r$ caused by a unit source at the source point $r_{0}$, then the field at $r$ caused by distribution $f\left(r_{0}\right)$ is the integral of $f\left(r_{0}\right) G\left(r, r_{0}\right)$ over the whole range of $r_{0}$ occupied by the course. The function $\mathrm{G}$ is called Green's function [3-5].

The Green's function is powerful tool of mathematical method which used I solving linear non-homogenous differential equation (ordinary and partial) [6-9].

\section{Preliminaries}

\section{Sturm-Liouville problem}

Consider a linear second order differential equation

$$
A(x) \frac{d^{2} y}{d x^{2}}+B(x) \frac{d y}{\partial x}+C(\mathrm{x}) \mathrm{y}+\lambda \mathrm{D}(\mathrm{x}) \mathrm{y}=0
$$

Where $\lambda$ is a parameter to be determined by the boundary conditions? $A(x)$ is positive continuous function, then by dividing every term by $A(x)$, equation (1) can be written as

$\frac{d^{2} y}{d x^{2}}+B(x) \frac{d y}{\partial x}+C(\mathrm{x}) \mathrm{y}+\lambda \mathrm{D}(\mathrm{x}) \mathrm{y}=0$

Where $b(\mathrm{x})=\frac{B(x)}{A(x)}, c(\mathrm{x})=\frac{C(x)}{A(x)}$ and $\mathrm{d}(\mathrm{x})=\frac{D(x)}{A(x)}$

Let us define integrating factor $p(x)$ by

$$
P(x)=\exp \left\{\int_{a}^{x} b(\xi) d \xi\right\}
$$

Multiplying equation (2) by $p(x)$, we have

$$
\begin{aligned}
& p(x) \frac{d^{2} y}{d x^{2}}+p(x) b(x) \frac{d y}{\partial x}+p(\mathrm{x}) \mathrm{c}(x) \mathrm{y}+\lambda p(x) d(\mathrm{x}) \mathrm{y}=0 \\
& \text { Since } \frac{d p(x)}{d x}=\frac{d}{d x}\left(e^{\int^{x} b(\zeta) d \zeta}\right)=e^{\int^{x} b(\zeta) d \zeta} \frac{d}{d x}\left(\int_{a}^{x} b(\zeta) \mathrm{d} \zeta\right)=p(x) b(x)
\end{aligned}
$$

So $\frac{d}{d x}\left[p(x) \frac{d y}{d x}\right]=p(x) \frac{d^{2} y}{d x^{2}}+\frac{d p(x)}{d x} \frac{d y}{d x}=p(x) \frac{d^{2} y}{d x^{2}}+p(\mathrm{x}) b(x) z$

Thus equation (3) can be written as

$\frac{d}{d x}\left[p(x) \frac{d y}{d x}\right]+q(x) y+\lambda r(x) y=0$

Where $q(x)=p(x) c(x)$ and $r(x)=p(x) d(x)$

Equation in form (4) is known as Sturm-Liouville equation. Satisfy the boundary conditions

Regular Sturm-Liouville Problem

In case $p(a) \neq 0$ and $p(b) \neq 0, p(x), q(x), r(x)$ are continuous, the Sturm-Liouville equation (4) can be expressed as

$$
L[y]=\lambda r(x) y
$$

Where $L=\frac{d}{d x}\left[p(x) \frac{d y}{d x}\right]+q(x)$

If the above equation is associated with the following boundary condition

$$
\begin{aligned}
& \alpha_{1} y(a)+\alpha_{2} y^{\prime}(a)=0 \\
& \beta_{1} y(b)+\beta_{2} y^{\prime}(b)=0
\end{aligned}
$$

Where $\alpha_{1}+\alpha_{2} \neq 0$ and $\beta_{1}+\beta_{2} \neq 0$

The equation (4) and the boundary condition (7) are called regular Sturm -Liouville problem (RSLP).

(a) Singular Sturm-Liouville problem

Consider the equation

$L[y]+\lambda r(x) y=0 a<x<b$

*Corresponding author: Abdelgabar Adam Hassana, Department of Mathematics, College of Science and Arts at Tabrjal, AlJouf University, Kingdom of Saudi Arabia Tel: +966 14624 7493; E-mail: jabra69b@yahoo.com

Received April 05, 2017; Accepted April 25, 2017; Published May 11, 2017

Citation: Hassana AA (2017) Green's Function Solution of Non-Homogenous Regular Sturm-Liouville Problem. J Appl Computat Math 6: 362. doi: 10.4172/21689679.1000362

Copyright: (c) 2017 Hassana AA. This is an open-access article distributed under the terms of the Creative Commons Attribution License, which permits unrestricted use, distribution, and reproduction in any medium, provided the original author and source are credited. 
Where $\mathrm{L}$ is defined by $(6), p(x)$ is smooth and $r(x)$ is positive, then the Sturm- Liouville problem is called singular if one of the following situations is occurred.

(i) If $p(a)=0$ or $p(b)=0$ or both

(ii) The interval $(\mathrm{a}, \mathrm{b})$ is infinite.

\section{(b)Eigenvalue and eigenfunction}

The Eigenvalue from equation (4) defining by a Sturm-Liouville operator can be expressed as

$$
\lambda=\frac{-1}{r(x)}\left[\frac{d}{d x}\left[p(x) \frac{d y}{d x}\right]+q(x)\right]
$$

The non-trival solutions that satisfy the equation and boundary conditions are called eigenfunctions. Therefore the eigenfunction of the Sturm-Liouville problem from complete sets of orthogonal bases for the function space is which the weight function is $r(x)$.

\section{The Dirac delta function}

The delta function is defined as

$$
\delta(x-\xi)= \begin{cases}0 & x \neq \xi \\ \infty & x=\xi\end{cases}
$$

But such that the integral of $\delta(x-\zeta)$ is normalized to unit

$$
\int_{-\infty}^{+\infty} \delta(x-\xi) d x=1
$$

In fact the first operator where Dirac used the delta function is the integration

$$
\int_{-\infty}^{+\infty} f(x) \delta(x-\xi) d x
$$

Where $f(x)$ is a continuous function, we have to find the value of the integration (12). Since $\delta(x \zeta)$ is zero for $x \neq \zeta$, the limit of integration may be change to $\zeta-\varepsilon$ and $\zeta+\varepsilon$, where $\varepsilon$ is a small positive number, $f(x)$ is continuous at $x-\zeta$, it's values within the interval $(\zeta-\varepsilon, \zeta-\varepsilon)$ will not different much from $f(\zeta)$, approximately that:

$$
\int_{-\infty}^{+\infty} f(x) \delta(x-\xi) d x=\int_{\xi-\infty}^{\xi+\infty} f(x) \delta(x-\xi) d x \approx \int_{\xi-\infty}^{\xi+\infty} f(\xi) \delta(x-\xi) d x
$$

With the approximation improving as $\varepsilon$ approaches zero.

From (11), we have

$$
I=\int_{-\infty}^{+\infty} \delta(x-\xi) d x=\int_{\xi-\infty}^{\xi+\infty} \delta(x-\xi) d x=1
$$

From all values of $\varepsilon$, then by letting $\varepsilon \rightarrow 0$, we can exactly have

$$
\int_{-\infty}^{+\infty} f(x) \delta(x-\xi) d x=f(\zeta)
$$

Despite the delta function considered as fundamental role in electrical engineering and quantum mechanics, but no conventional could be found that satisfies (10) and (11), then the delta function sought to be view as the limit of the sequence of strongly peaked function $\delta_{n}(x)$ such that

$$
\delta(x)=\lim _{n \rightarrow \infty} \delta_{n}(x)
$$

$$
\left.\begin{array}{l}
\delta_{n}(x)=\frac{n}{\pi\left(1+n^{2} x^{2}\right)} \\
\delta_{n}(x)=\frac{n}{\sqrt{\pi}} e^{-n^{2} x^{2}} \\
\delta_{n}(x)=\frac{\sin ^{2}(n x)}{n \pi x^{2}} e t c
\end{array}\right\}
$$

(c) Some important properties of Dirac delta function

\section{Property (1): Symmetry}

$\delta(-x)=\delta(x)$

Proof

Let $\zeta=-x$, then $d x=-d \zeta$

We can write:

$\int_{-\infty}^{+\infty} f(x) \delta(-x) d x=\int_{+\infty}^{-\infty} f(\zeta) \delta(\zeta) d \zeta \approx \int_{-\infty}^{+\infty} f(\zeta) \delta(\zeta) d \zeta=f(0)$

But, $\int_{-\infty}^{+\infty} f(x) \delta(x) d x=f(0)$

Therefore, from (19) and (20), we conclude that $\delta(-x)=\delta(x)$

Property (2): Scaling

$\delta(a x)=\frac{1}{|a|} \delta(x)$
Proof

Let $\zeta=$ ax, then $d x=\frac{1}{a} d \zeta$

If $a>0$, then

$\int_{-\infty}^{+\infty} f(x) \delta(a x) d x=\int_{+\infty}^{-\infty} f\left(\frac{\zeta}{a}\right) \delta(\zeta) \frac{1}{a} d \zeta=\frac{1}{a} \int_{-\infty}^{+\infty} f\left(\frac{\zeta}{a}\right) \delta(\zeta) d \zeta=\frac{1}{a} f\left(\frac{0}{a}\right) f(0)$

Since $\int_{-\infty}^{+\infty} f(\zeta) \frac{1}{a} \delta(x) d x=\frac{1}{a} \int_{-\infty}^{+\infty} f(x) \delta(x) d x=\left(\frac{1}{a}\right) f(0)$

Therefore:

$\delta(a x)=\frac{1}{a} \delta(x)$

Similarly for $a<0$,

$\delta(a x)=\frac{1}{-a} \delta(x)$

then

We can write:

$\delta(a x)=\frac{1}{|a|} \delta(x)$

Property (3)

$\delta\left(x^{2}-a^{2}\right)=\frac{1}{|2 a|}[\delta(x+a)+\delta(x-a)]$

Proof

The argument of this function goes to zero when $x=a$ and $x=-a$, wherefore

$\int_{-\infty}^{+\infty} f(\zeta) \delta\left(x^{2}-a^{2}\right) d x=\int_{-\infty}^{+\infty} f(x)[\delta(x+a)+\delta(x-a)] d x$

Only at the zero of the argument of the delta function that is: 


$$
\int_{-\infty}^{+\infty} f(\zeta) \delta\left(x^{2}-a^{2}\right) d x=\int_{-a-e}^{-a+e} f(x)\left[\delta\left(x^{2}-a^{2}\right) d x+\int_{a-e}^{a+e} f(x) \delta\left(x^{2}-a^{2}\right)\right] d x
$$

Near the two zeros $x^{2}-a^{2}$ can be approximated as:

$$
\left(x^{2}-a^{2}\right)=(x-a)(x+a)= \begin{cases}(-2 a)(x+a), & x \rightarrow-a \\ (+2 a)(x-a), & x \rightarrow+a\end{cases}
$$

In the limit as $\varepsilon \rightarrow 0$ the integral (23) becomes:

$$
\begin{aligned}
\int_{-\infty}^{+\infty} f(\zeta) \delta\left(x^{2}-a^{2}\right) & d x=\int_{-a-e}^{-a+e} f(\mathrm{x}) \delta[(-2 a)(x+a)] d x+\int_{a-e}^{a+e} f(\mathrm{x}) \delta[(2 a)(x-a)] d x \\
& =\frac{1}{|2 a|} \int_{a-e}^{a+e} f(\mathrm{x}) \delta[(x) \delta(x+a)]+\frac{1}{|2 a|} \int_{a-e}^{a+e} f(\mathrm{x}) \delta(x-a) d x \\
& =\int_{-\infty}^{+\infty} f(x) \frac{1}{|2 a|}[\delta[x-a]+\delta(x-a)] d x
\end{aligned}
$$

Therefore: $\delta\left(x^{2}-a^{2}\right)==\frac{1}{|2 a|}[\delta[x-a]+\delta(x-a)]$

\section{Green's Function}

\section{The concept of Green's function}

In the case of ordinary differential equation we can express this problem as

\section{$L[y]=f$}

Where $L$ is a linear differential operation $f(x)$ is known function and $y(x)$ is desired solution. We will show that the solution $y(x)$ is given by an integral involving that Green's function $G(x, \xi)$.

\section{Green's function for ordinary differential equation}

Here we consider non-homogenous ordinary differential equation

$L[y]=f$

Where $\mathrm{L}$ is an ordinary linear differential operator that can be represented bySturm-Liouville operator, i.e.

$$
L=\frac{d}{d x}\left[p(x) \frac{d y}{d x}\right]+q(x)
$$

And the Sturm-Liouville type is gives by

$$
\frac{d}{d x}\left[p(x) \frac{d y}{d x}\right]+q(x) y+\lambda r(x) y=-f(x)
$$

Where $\lambda$ is a parameter. Now consider the linear non homogenous ordinary differential equation of the form

$$
\frac{d}{d x}\left[p(x) \frac{d y}{d x}\right]+q(x) y=-f(x) a \leq x \leq b
$$

With the boundary condition

$$
\left.\begin{array}{l}
\alpha_{1} y(a)+\alpha_{2} y^{\prime}(a)=0 \\
\beta_{1} y(b)+\beta_{2} y^{\prime}(b)=0
\end{array}\right\}
$$

where the constant are such that $\alpha_{1}+\alpha_{2} 0$ and ${ }_{1}+\beta_{2} \neq 0$ if $\lambda=0$ then equation (27) and equation (28) are identical in the interval and $r(x)$ are real and positive in that interval.

Now we are seeking to determine the Green's function $G$ for the equation satisfies the following

$$
\frac{d}{d x}\left[p(x) \frac{d G}{d x}\right]+q(x) y=-\delta(x-\zeta)
$$

With the boundary condition

$$
\begin{aligned}
& \alpha_{1} G(a)+\alpha_{2} G^{\prime}(a, \zeta)=0 \\
& \beta_{1} G(b)+\beta_{2} G^{\prime}(b, \zeta)=0
\end{aligned}
$$

Now consider the region $a \leq x<\zeta$.

Let $y_{1}(x)$ be a nontrivial solution at $x=a$, i.e

$\alpha_{1} y_{1}(a)+\alpha_{2} y^{\prime}(a)=0$

Then $\alpha_{1} y_{1}(a, \zeta)+\alpha_{2} G^{\prime}(a, \zeta)=0$

The wronskian of $y_{1}$ and $G$ must vanish at $x=a$ or

$y_{1}(a) G^{\prime}(a, \zeta)+y_{1}^{\prime}(a) G(a, \zeta)=0$

So $G(x, \zeta)=u_{1} y_{1}(x)$ for $a \leq x<\zeta$

Where $u_{1}$ is an arbitrary constant. Similarly if the nontrivial solution $y_{2}(x)$ satisfies the homogeneous equation and the condition at $x=b$, then

$G(x, \zeta)=u_{2} y_{2}(x)$ for $\zeta \leq x<b$

Now by integrating equation (29) from $\zeta-\varepsilon$ to $\zeta+\varepsilon$ we obtain

$$
P(x) \frac{d G(x, \zeta)}{d x}\left[{ }_{\zeta+\varepsilon}^{\zeta+\varepsilon}+\int_{\zeta-\varepsilon}^{\zeta+\varepsilon} q(\mathrm{x}) \mathrm{G}(x, \zeta) d x=-1\right]
$$

Since $G(x, \zeta)$ and $q(x)$ are continuous at $x=\zeta$ then we have

$$
\frac{d G(\bar{\zeta}, \zeta)}{d x}=\frac{d G(\bar{\zeta}, \zeta)}{d x}=\frac{1}{p(\zeta)}
$$

The continuity condition of $\mathrm{G}$ and the Jump discontinuity of $G^{\prime}$ at $x=\zeta$ from equation (33) , (34) and equation (36) imply

$$
\left.\begin{array}{l}
u_{1} y_{1}(\zeta)-u_{2} y_{2}=0 \\
u_{1} y_{1}(\zeta)-u_{2} y_{2}^{\prime}=\frac{1}{p(\zeta)}
\end{array}\right\}
$$

we can solve equation (37) for $u_{1}$ and $u_{2}$ provided the wronskian $y_{1}$ and $y_{2}$ doesn't varnish at $x=\zeta$ or

$$
\begin{aligned}
& y_{1}(\zeta) y_{2}^{\prime}(\zeta) y_{1}^{\prime}(\zeta) \neq 0 \\
& \text { i.e., } w(\zeta)=\left|\begin{array}{ll}
y_{1}(\zeta) & y_{1}(\zeta) \\
y_{1}(\zeta) & y_{2}{ }^{\prime}(\zeta)
\end{array}\right| \neq 0
\end{aligned}
$$

The system of equation (37) has the solution

$$
\begin{aligned}
& u_{1}=\frac{-y_{2}(\zeta)}{p(\zeta) w(\zeta)} \\
& \text { and } \\
& u_{2}=\frac{-y_{1}(\zeta)}{p(\zeta) w(\zeta)}
\end{aligned}
$$

Where $w(\zeta)$ is the wronskian of $y_{1}(x)$ and $y_{2}(x)$ at $x=\zeta$

Therefore

$$
\begin{aligned}
G(x, \zeta) & =\frac{y_{1}(x<) y_{2}(x>)}{p(\zeta) w(\zeta)} \\
G(x, \zeta) & =\left\{\begin{array}{l}
\frac{y_{1}(x) y_{2}(\zeta)}{p(\zeta) w(\zeta)}, x<\zeta \leq b \\
-\frac{y_{1}(x<) y_{2}(x>)}{p(\zeta) w(\zeta)}, a<\zeta \leq x
\end{array}\right\}
\end{aligned}
$$


Now from (42) the solution (27) can be expressed as

$y(\mathrm{x})=\int_{a}^{b} G(x, \zeta) f(\zeta) d \zeta$

So $y(\mathrm{x})=\int_{a}^{x} G(x, \zeta) f(\zeta) d \zeta+\int_{a}^{b} G(x, \zeta) f(\zeta) d \zeta$

Some properties of Green's function:

The following properties followed Green's function

Property (i)

$G(x, \zeta)$ is exit because both $p(x) \neq 0, w(x) \neq 0$

\section{Proof}

From equation (32) and (33) we obtain

$u_{1}=\frac{u_{1} y_{2}(x)}{y_{1}(x)}$

And from equation (35) at $x=\zeta$ we have

$u_{1} y_{1}^{\prime}(x)-u_{2} y_{2}^{\prime}(x)=-\frac{1}{p(x)}$

Substituting from (44) into (45) we obtain

$u_{1}\left[y_{2}(x) y_{1}^{\prime}(x)-y_{1}(x) y_{2}^{\prime}(x)\right]=-\frac{y_{1}(x)}{p(x)}$

Let $w(x)=y_{1}(x) y_{2}(x)-y_{2}(x) y_{1}(x)$

i.e. $w(x)=\left|\begin{array}{lc}y_{1}(x) & y_{2}(x) \\ y_{1}(x) & y_{2}^{\prime}(x)\end{array}\right|$

$u_{1}=-\frac{-y_{2}(x)}{p(x) w(x)}$

and

$u_{2}=-\frac{-y_{1}(x)}{p(x) w(x)}$

but $u_{1}$ and $u_{2}$ are arbitrary constant

$\therefore$ both $p(x) \neq 0$ and $w(x) \neq 0$

Property (ii)

$G(x, \zeta)$ satisfies the homogenous equation except at $x=\zeta$

\section{Proof}

From equation (37) $\delta(x-\zeta)=0$ except at $x=\zeta$

$p^{\prime}(x) G^{\prime}(x, \zeta)+q(x) G(x, \zeta)=0$

where $p(x) \neq 0, p^{\prime}(x), q(x)$ are continuous on $[\mathrm{a}, \mathrm{b}]$

Property (iii)

$G(x, \zeta)$ is continuous at $x=\zeta$

\section{Proof}

$\lim _{x \rightarrow \zeta} G(x, \zeta)=y_{2}(\zeta)$

$\lim _{x \rightarrow \zeta} G(x, \zeta)=y_{1}(\zeta)$

Therefore $G(x, \zeta)$ is continuous at $x=\zeta$

\section{Property (iv)}

The first and second derivatives are continuous for all $x \neq \zeta$ in $a \leq x, \zeta \leq b$

\section{Proof}

Differentiation equation (32) with respect to $x$

$$
\left.\begin{array}{rl}
\frac{d}{d x} \int_{a}^{b} G(x, \zeta) f(\zeta) d(\zeta)= & \int_{a}^{x} \frac{d^{2} G(x, \zeta)}{d x} f(\zeta) d \zeta+\frac{d G\left(x, x^{-}\right)}{d x} d(\zeta) \\
& +\int_{x}^{b} \frac{d^{2} G(x, \zeta)}{d x} f(\zeta) d \zeta-\frac{d G\left(x, x^{+}\right)}{d x} d(\zeta)
\end{array}\right\}
$$

But $\mathrm{G}(\mathrm{x}, \zeta)$ is continuous everywhere, there we have $G\left(x, x^{+}\right)=G\left(x, x^{-}\right)$so that

$\frac{d}{d x} \int_{a}^{b} G(x, \zeta) f(\zeta) d(\zeta)=\int_{a}^{x} \frac{d G(x, \zeta)}{d x} f(\zeta) d \zeta+\int_{x}^{b} \frac{d G(x, \zeta)}{d x} f(\zeta) d \zeta$

Differentiation once more gives

$$
\left.\begin{array}{rl}
\frac{d^{2}}{d x^{2}} \int_{a}^{b} G(x, \zeta) f(\zeta) d(\zeta)= & \int_{a}^{x} \frac{d^{2} G(x, \zeta)}{d x^{2}} f(\zeta) d \zeta+\frac{d G\left(x, x^{-}\right)}{d x} d \zeta \\
& +\int_{b}^{b} \frac{d^{2} G(x, \zeta)}{d x^{2}} f(\zeta) d \zeta-\frac{d G\left(x, x^{+}\right)}{d x} d \zeta
\end{array}\right\}
$$

The second and fourth terms on the right side will not cancel in this case to the contrary

$$
\frac{d G\left(x, x^{-}\right)}{d x}-\frac{d G\left(x, x^{+}\right)}{d x}=\frac{1}{p(\zeta)}
$$

We note that the term $\frac{d G\left(x, x^{-}\right)}{d x}$ denotes a differentiation of $G(x, \zeta)$ with respect to $x$ using the $x>\zeta$, at $\zeta \rightarrow x$. Thus

$$
\frac{d G\left(x, x^{-}\right)}{d x}=\lim _{\substack{\zeta \rightarrow X \\ \zeta \rightarrow \infty}} \frac{y_{1}^{\prime}(x) y_{1}(\zeta)}{p(\zeta) w(\zeta)}=\frac{y_{1}^{\prime}(x) y_{1}(\zeta)}{p(x) w(\zeta)}
$$

For: $d G\left(x, x^{+}\right)$we use the $x<\zeta$ then

$\frac{d G\left(x, x^{+}\right)}{d x}=\lim _{\substack{\zeta \rightarrow X \\ \zeta \rightarrow \infty}} \frac{y_{1}^{\prime}(x) y_{2}(\zeta)}{p(\zeta) w(\zeta)}=\frac{y_{1}^{\prime}(x) y_{2}(\zeta)}{p(x) w(\zeta)}$.

Property (v)

$G(x, \zeta)$ is symmetric in $x$ and $\zeta$

Poof

Let $G(x, \zeta)=\left\{\begin{array}{l}\frac{-y_{1}^{\prime}(x) y_{2}(\zeta)}{p(\zeta) w(\zeta)} \\ \frac{-y_{2}^{\prime}(x) y_{1}(\zeta)}{p(\zeta) w(\zeta)}\end{array} \quad a \leq x<\zeta\right.$

$\lim _{x+\xi} G\left(x^{+}, \zeta\right)=\frac{y_{2}^{\prime}(x) y_{1}(\zeta)}{p(\zeta) w(\zeta)}$

and $\lim _{x+\xi} G\left(x^{+}, \zeta\right)=\frac{y_{2}^{\prime}(x) y_{1}(\zeta)}{p(\zeta) w(\zeta)}$

Problem(1)

Find the solution of the following problem by construction the Green's function

$y^{\prime \prime}+k^{2} y=-f(x), 0<x<\mathrm{L}$

Subject to the boundary conditions

$y(0)+y(L)=0$ 


\section{With $k \neq 0$}

\section{Solution}

Let $G(x, \zeta)$ be the Green's function of the problem, then

$G^{\prime \prime}+k^{2} G=-\delta(x \zeta)$

With $G(0, \zeta)+G(L, \zeta)=0$

The general solution to the homogenous equation is given by

$y(x)=c_{1} \cos k x+c_{2} \sin k x$

Now applying to the above solution

$y(0)=0$

$y(0)=\mathrm{c}_{1} \cos k x+c_{2}(0)=0 \Rightarrow c_{1}=0$

$\therefore y_{1}(x)=c_{2} \sin k x$

and $y(L)=0 \Rightarrow 0=c_{1} \cos L+c_{2} \sin k L$

$\therefore c_{1}=-\frac{c_{2} \sin k L}{\cos L}$

$y_{2}(x)=\frac{c_{2}}{\cos k \mathrm{~L}}(\cos k x \sin k L-\sin k x \cos k L)=\frac{C_{2}}{\operatorname{Cos} k L} \sin \mathrm{k}(\mathrm{L}-x)$

The Wronskian is given by

$w(x)=\left|\begin{array}{ll}c_{2} \sin k x & \frac{C_{2}}{\operatorname{Cos} k L}(\mathrm{~L}-x) \\ c_{2} k \cos k x & \frac{C_{2} k}{\operatorname{Cos} k L} \cos (\mathrm{L}-x)\end{array}\right|=\frac{C_{2}}{\operatorname{Cos} k L} \sin k \mathrm{~L}$

The Green's function is given by

$G(x, \zeta)= \begin{cases}\frac{y_{1}(x) y_{2}(\zeta)}{p(\zeta) w(\zeta)} & 0 \leq x<\zeta \\ \frac{y_{2}(x) y_{1}(\zeta)}{p(\zeta) w(\zeta)} & \zeta<x \leq L\end{cases}$
$G(x, \zeta)= \begin{cases}-\frac{\sin k x \sin k(L-\zeta)}{k \sin k L} & 0 \leq x<\zeta \\ -\frac{\sin k(L-x) \sin k \zeta}{k \sin k L} & \zeta<x \leq L\end{cases}$

The solution is given by

$$
\begin{aligned}
y(\mathrm{x}) & =\int_{a}^{L} G(x, \zeta) f(\zeta) d \zeta \\
y(\mathrm{x}) & =\int_{0}^{X} \frac{\sin k x \sin l(L-\zeta)}{k \sin k L} f(\zeta) d \zeta \\
& -\int_{x}^{l} \frac{\sin k(L-x) \sin k \zeta}{k \sin k L} f(\zeta) d \zeta
\end{aligned}
$$

\section{Problem (2)}

Solve the problem by construction the Green's function $y^{\prime \prime}-\frac{1}{a}=-f(x), 0<x<l$

with the boundary condition

$y(0)+y(L)=0$ where $a \neq 0$

\section{Solution}

Let $G(x, \zeta)$ be the Green's function of the problem, then
$G^{\prime \prime}-\frac{1}{a}=-\delta(x-\zeta)$

With the boundary condition $G(0, \zeta)+G(L, \zeta)=0$

The general solution to the homogenous equation is given by

$Y(x)=\frac{1}{2 a}+c_{1} x^{2}+c_{2}$

Applying to the above solution

then, and $y(0)=0$ then $c_{2}=0$, and

$\therefore y_{1}(x)=\frac{x^{2}}{2 a}+c_{1} x$

Applying the boundary condition, then $y(L)=0 \quad c_{2}=\left(\frac{L^{2}}{2 a}+c_{1} L\right)$, and

$y_{2}(x)=-\frac{c_{2}}{2 a}\left(x^{2}-L^{2}\right)+c_{1}(x-L)$

The Wronskian is given by

$w(x)=L\left(\frac{x}{a}+c_{1}\right)\left(\frac{L}{2 a}+c_{1}\right)$

The Green's function is given by

$$
\begin{aligned}
& G(x, \zeta)=\left\{\begin{array}{l}
\frac{y_{1}^{\prime}(x) y_{2}(\zeta)}{p(\zeta) w(\zeta)} \\
\frac{y_{2}(x) y_{1}(\zeta)}{p(\zeta) w(\zeta)}
\end{array} \quad 0 \leq x<\zeta\right. \\
& G(x, \zeta)= \begin{cases}\frac{\left(\frac{x^{2}}{2 a}+c_{1} x\right)\left[\frac{1}{2 a}\left(\zeta^{2}-L^{2}\right)+c_{1}(\zeta-L)\right]}{L\left(\frac{x}{2 a}+c_{1}\right)\left(\frac{L}{2 a}+c_{1}\right)} & 0 \leq x<\zeta \\
\frac{\left(\frac{x^{2}}{2 a}+c_{1} x\right)\left[\frac{1}{2 a}\left(\zeta^{2}-L^{2}\right)+c_{1}(\zeta-L)\right]}{L\left(\frac{x}{2 a}+c_{1}\right)\left(\frac{L}{2 a}+c_{1}\right)} & \zeta<x \leq L\end{cases}
\end{aligned}
$$

Where we chose $c_{1}=1$, then

$$
G(x, \zeta)= \begin{cases}\frac{x\left(\frac{x}{2 a}+1\right)(\zeta-L)\left[\frac{1}{2 a}(\zeta+L)+c_{1}(\zeta-L)\right]}{L\left(\frac{x}{2 a}+c_{1}\right)\left(\frac{L}{2 a}+c_{1}\right)} & 0 \leq x<\zeta \\ \frac{\left(\frac{x^{2}}{2 a}+c_{1} x\right)\left[\frac{1}{2 a}\left(\zeta^{2}-L^{2}\right)+c_{1}(\zeta-L)\right]}{L\left(\frac{x}{2 a}+c_{1}\right)\left(\frac{L}{2 a}+c_{1}\right)} & \quad \zeta<x \leq L\end{cases}
$$

The solution is given by

$$
\begin{aligned}
& y(x)=\int_{a}^{L} G(x, \zeta) f(\zeta) d \zeta \\
& y(x)=\int_{a}^{x} \frac{x\left(\frac{x}{2 a}+1\right)(\zeta-L)\left[\frac{1}{2 a}(\zeta+L)+1\right]}{L\left(\frac{x}{2 a}+1\right)\left(\frac{L}{2 a}+1\right)} f(\zeta) d \zeta \\
& +\int_{x}^{L} \frac{\frac{1}{2 a}(x-L)\left[\zeta\left(\frac{1}{2 a}+1\right)+\frac{1}{2 a}(x+L)\right]}{L\left(\frac{x}{a}+1\right)\left(\frac{L}{2 a}+1\right)} f(\zeta) d \zeta
\end{aligned}
$$


Citation: Hassana AA (2017) Green's Function Solution of Non-Homogenous Regular Sturm-Liouville Problem. J Appl Computat Math 6: 362 . doi: $10.4172 / 2168-9679.1000362$

Page 6 of 6

\section{References}

1. Gwaiz MA Al (2008) Sturm-Liouville theory\& its Application. Verlag London limited.

2. Blakledge GE, Yardley JP (2000) Analytic methods for Partial differential Equations. Sprenger London Limited.

3. Tintang K (2007) Mathematical methods for Engineers and Scientists 3.

4. Gerald BH (1992) Fourier analysis and Application. Wodsworth. Inc. Belmon California.
5. Tintang K (2007) Mathematical methods for Engineers and Scientists 2.Verlag Berlin Heidelbarg.

6. Neta B (2002) Partial differential equations, lecture notes, Naval postgraduate School. California.

7. Pinchover Y, Rubinstein J (2005) An introduction to Partial differential equations. Cambridge University.

8. Roach GF (1970) Green's function introductory theory with Application. New York Toronto Melbourne.

9. Addison W (1984) Boundary Value problem, Monterey California. 